\title{
Attenuating the singularities of plurisubharmonic functions
}

\author{
by Christer O. Kiselman (Uppsala)
}

\begin{abstract}
Resumo (Malfortigi la malregulejojn la plursubharmonaj funkcioj). Ni studas la malregulejojn de la plursubharmonaj funkcioj per metodoj de la konveksa analitiko. Teoremoj pri analitikeco de rafinita nombro de Lelong estas pruvitaj.
\end{abstract}

Abstract. We study the singularities of plurisubharmonic functions using methods from convexity theory. Analyticity theorems for a refined Lelong number are proved.

1. Introduction. The plurisubharmonic functions, which appear in complex analysis first as logarithms of moduli of holomorphic functions and as analogues of potentials, are useful for many constructions since they are easier to manipulate than holomorphic functions - this is why Lelong [1985] includes them among "les objets souples de l'analyse complexe." The present paper is written in this spirit: we show how to construct in a straightforward way new plurisubharmonic functions from old ones using standard methods of convex analysis. These new functions can then be used to find analytic varieties that are connected with the original function, or rather with its singularities. We shall therefore first describe how one can measure the singularity of a plurisubharmonic function: this is done using the Lelong number and the integrability index.

The Lelong number measures how big (or "heavy") the singularities of a plurisubharmonic function are. It generalizes the notion of multiplicity of a zero of a holomorphic function. To define it, we first form the measure $\mu=(2 \pi)^{-1} \Delta f$, where $\Delta$ is the Laplacian in all $2 n$ real variables $\operatorname{Re} z_{j}$, $\operatorname{Im} z_{j}$. Note that when $f=\log |h|$ is the logarithm of the absolute value of a holomorphic function of one variable, then $\mu$ is a sum of point masses, one at each zero of $h$ and with weight equal to the multiplicity of the zero. The Lelong number of $f$ at a point $x$ is by definition the $(2 n-2)$-dimensional

1991 Mathematics Subject Classification: Primary 32F05, 32B15; Secondary 52A41.

Key words and phrases: plurisubharmonic function, analytic variety, convex function, Fenchel transformation.

Research partially supported by the Swedish Natural Science Research Council. 
density of the measure $\mu$ at $x$. More explicitly, it is the limit as $r \rightarrow 0$ of the mean density of $\mu$ in the ball of center $x$ and radius $r$ :

$$
\nu_{f}(x)=\lim _{r \rightarrow 0} \frac{\mu(x+r B)}{\lambda_{2 n-2}\left(r B \cap \mathbb{C}^{n-1}\right)},
$$

where $\lambda_{k}$ denotes $k$-dimensional Lebesgue measure. Note that we compare the mass of $\mu$ in the ball $x+r B$ with the volume of the ball of the same radius in $\mathbb{C}^{n-1}$, i.e., of real dimension $2 n-2$. This makes sense, because if $f=\log |h|$ with $h$ holomorphic, then $\mu$ is a mass distribution on the $(2 n-2)$ dimensional zero set of $h$. If $n=1$, then $\lambda_{2 n-2}\left(r B \cap \mathbb{C}^{n-1}\right)=\lambda_{0}(\{0\})=1$, and $\nu_{f}(x)$ is just the mass of $\mu$ at $x$.

A plurisubharmonic function $f$ is often approximated by $f_{j}=\max (-j, f)$ or by smooth functions $f_{j}=f * \psi_{j}$ obtained by convolution. However, in these cases the functions $f_{j}$ never take the value $-\infty$, so their Lelong numbers $\nu_{f_{j}}(x)$ are zero everywhere; their singularities as measured by the Lelong number do not approach those of $f$ as $j \rightarrow+\infty$. Here we shall construct functions $f_{\tau}$ depending on a non-negative number $\tau$ such that $f_{0}=f$ and $f_{\tau}$ has Lelong number $\nu_{f_{\tau}}(x)=\left(\nu_{f}(x)-\tau\right)^{+}$. It turns out that the family $\left(f_{\tau}\right)_{\tau}$ can be used in various constructions. The singularities of $f_{\tau}$ are the same as those of $f$ but attenuated in a certain sense. More precisely, the important property is that $\nu_{f_{\tau}}(x)>0$ if $\tau<\nu_{f}(x)$, whereas the singularity is completely killed, i.e., $\nu_{f_{\tau}}(x)=0$, if $\tau>\nu_{f}(x)$. In this context it is convenient to define the Lelong number of a family of plurisubharmonic functions. We prove analyticity theorems for the superlevel sets of such numbers; see Section 4.

If $f$ is plurisubharmonic and $t$ a positive number, the function $\exp (-f / t)$ may or may not be integrable. The set of all $t$ such that this function is locally integrable in the neighborhood of a certain point is an interval, and its endpoint measures how singular $f$ is. This is the reason behind the integrability index $\iota_{f}$ to be defined in Section 3 (see (3.3)). From the HörmanderBombieri theorem we get analyticity theorems for the integrability index (see (3.4)). There is a relation between the integrability index and the Lelong number: $\iota_{f} \leq \nu_{f} \leq n \iota_{f}$, where $n$ is the complex dimension of the space; see Theorem 3.5. This relation cannot be improved (see Example 3.6), but nevertheless it will suffice to yield analyticity theorems for the Lelong number. The reason for this is, roughly speaking, that if we subtract the same quantity $\tau$ from two numbers like $\nu_{f}(x)$ and $\nu_{f}\left(x^{\prime}\right)$ with $\nu_{f}\left(x^{\prime}\right)>\nu_{f}(x)>\tau$, then the quotient between $\nu_{f}\left(x^{\prime}\right)-\tau$ and $\nu_{f}(x)-\tau$ can be large, for instance larger than the dimension $n$. This is why analyticity theorems for sets of plurisubharmonic functions are useful when it comes to proving analyticity theorems for a single function.

We also consider a refined Lelong number, first presented at a seminar in Tunis on March 6, 1986 (see Kiselman [1987]). This number, denoted by 
$\nu_{f}(x, y)$ and defined in Section 5, depends on a vector $y$ and is a concave function of that vector. The methods of convex analysis can therefore be applied to study it. For any fixed $y$, it is a special case of Demailly's generalized Lelong number [1987]. For related studies of Lelong numbers, see Abrahamsson [1988] and Wang [1991].

2. Spherical means and spherical suprema. Let $f$ and $q$ be two given plurisubharmonic functions in an open set $\omega$ in $\mathbb{C}^{n}$; we write this as $f, q \in \operatorname{PSH}(\omega)$. We define an open set $\Omega_{q}$ in $\mathbb{C}^{n} \times \mathbb{C}$ as

$$
\Omega_{q}=\{(x, t) \in \omega \times \mathbb{C}: q(x)+\operatorname{Re} t<0\},
$$

and we note immediately that $\Omega_{q}$ is pseudoconvex if $\omega$ is pseudoconvex, for the function $(x, t) \mapsto q(x)+\operatorname{Re} t$ is plurisubharmonic in $\omega \times \mathbb{C}$. We assume that $q(x) \geq-\log d_{\omega}(x)$ for all $x \in \omega$, denoting by $d_{\omega}(x)$ the Euclidean distance from $x$ to the complement of $\omega$, and we note that then $(x, t) \in \Omega_{q}$ implies that the closed ball of center $x$ and radius $\left|e^{t}\right|$ is contained in $\omega$. We define two functions $u$ and $U$ in $\Omega_{q}$ by putting

$$
\begin{aligned}
& u(x, t)=u_{f}(x, t)=u_{f, q}(x, t)=f_{z \in S} f\left(x+e^{t} z\right), \quad(x, t) \in \Omega_{q} \\
& U(x, t)=U_{f}(x, t)=U_{f, q}(x, t)=\sup _{z \in S} f\left(x+e^{t} z\right), \quad(x, t) \in \Omega_{q} .
\end{aligned}
$$

Here $S$ is the Euclidean unit sphere, and the barred integral sign indicates the mean value: in general,

$$
f_{A} f=\int_{A} f / \int_{A} 1 \quad \text { provided } 0<\int_{A} 1<+\infty .
$$

So $u_{f}(x, t)$ is the mean value of $f$ over the sphere $x+e^{t} S$, and $U_{f}(x, t)$ is the supremum of $f$ over the same sphere. Since we usually keep $q$ fixed, the dependence on that function need not always be shown. If $\omega \neq \mathbb{C}^{n}$, the simplest choice of $q$ is just $q=-\log d_{\omega}$. Then $q>-\infty$ everywhere. However, if $\omega=\mathbb{C}^{n}$, then it is usually not convenient to use $q=-\log d_{\omega}=-\infty$, because with this choice of $q$, the behavior of $f$ at infinity would influence the local properties of the functions we construct. In this case it is best just to take $q=0$.

Thanks to our assumption $\exp (-q(x)) \leq d_{\omega}(x)$, the functions $u_{f, q}$ and $U_{f, q}$ are well defined and do not take the value $+\infty$ in $\Omega_{q}$. Moreover, by standard results, $u_{f, q}, U_{f, q} \in \operatorname{PSH}\left(\Omega_{q}\right)$. We define them to be $+\infty$ outside $\Omega_{q}$.

Clearly, $u_{f} \leq U_{f}$, and we shall see that there are inequalities in the opposite direction. We can note quickly that $u_{a f+b g}=a u_{f}+b u_{g}$ for non-negative $a, b$, even for real $a, b$, which implies that the function $u_{f}$ depends linearly of $f$ in the linear space of all Borel measurable functions which are integrable on spheres, thus in particular on the space $\delta \mathrm{PSH}(\omega)$ of delta-plurisubharmonic 
functions, i.e., the vector space spanned by those plurisubharmonic functions which are not identically $-\infty$ in any component of $\omega$. We shall see that this implies that the Lelong number is a linear function on $\delta \operatorname{PSH}(\omega)$. As for the function $U_{f}$, we can only say that $U_{a f+b g} \leq a U_{f}+b U_{g}$ for $a, b \geq 0$, which implies that $U_{f}$ is a convex function of $f$, and the Lelong number a concave function of $f$. But when it comes to the maximum of two functions, we have $U_{\max (f, g)}=\max \left(U_{f}, U_{g}\right)$, which implies that $\nu_{\max (f, g)}=\min \left(\nu_{f}, \nu_{g}\right)$, whereas for the mean we can only say that $u_{\max (f, g)} \geq \max \left(u_{f}, u_{g}\right)$, which implies that $\nu_{\max (f, g)} \leq \min \left(\nu_{f}, \nu_{g}\right)$. It is therefore useful to know that the Lelong number can be defined by either $u_{f}$ or $U_{f}$, because this enables us to use the best properties of either one.

We can define the Lelong number as the slope at $-\infty$ of the function $t \mapsto u(x, t)$. As a consequence of the maximum principle, $u(x, t)$ and $U(x, t)$ are increasing in $t$; by Hadamard's three-circle theorem, they are convex functions of $t$. Therefore their slopes at $-\infty$ exist:

$$
\nu_{f}(x)=\lim _{t \rightarrow-\infty} \frac{u(x, t)}{t} \text { and } N_{f}(x)=\lim _{t \rightarrow-\infty} \frac{U(x, t)}{t}
$$

both exist. This follows from the fact that the slopes

$$
\frac{u(x, t)-u\left(x, t_{0}\right)}{t-t_{0}} \text { and } \frac{U(x, t)-U\left(x, t_{0}\right)}{t-t_{0}}
$$

are increasing in $t$. The first limit $\nu_{f}(x)$ is the Lelong number of $f$ at $x$, and the definition we shall use in this paper. The definition (1.1) of the Lelong number as the density of a measure is equivalent to (2.4) as can be proved without difficulty using Stokes' theorem; see Kiselman [1979].

Since $u_{f} \leq U_{f}$, we have $\nu_{f}(x) \geq N_{f}(x)$. We shall now see that the two numbers are equal. To this end we shall use Harnack's inequality, which takes the form

$$
\frac{1+|x| / r}{(1-|x| / r)^{m-1}} h(0) \leq h(x) \leq \frac{1-|x| / r}{(1+|x| / r)^{m-1}} h(0)
$$

for harmonic functions which satisfy $h \leq 0$ in the ball of radius $r$ in $\mathbb{R}^{m}$. If $f$ is subharmonic in a neighborhood of the closed ball $e^{s} B$ in $\mathbb{C}^{n}$, we can consider its harmonic majorant $h$ there, which satisfies $f(x) \leq h(x)$ and

$$
h(0)=f_{z \in S} h\left(e^{s} z\right)=f_{z \in S} f\left(e^{s} z\right)=u(0, s) .
$$

Therefore

$$
U(0, t)=\sup _{e^{t} S} f \leq \sup _{e^{t} S} h \leq \frac{1-e^{t-s}}{\left(1+e^{t-s}\right)^{2 n-1}} u(0, s), \quad t<s,
$$

provided $f \leq 0$ in $e^{s} B$. If we apply this inequality to the function $f-U(0, s)$, which is non-positive in $e^{s} B$ by definition, we get, writing $U(t)$ instead of 
$U(0, t)$ for simplicity,

$$
U(t)-U(s) \leq \frac{1-e^{t-s}}{\left(1+e^{t-s}\right)^{2 n-1}}(u(s)-U(s)),
$$

or equivalently,

$$
U(t) \leq\left(1-\lambda_{t-s}\right) U(s)+\lambda_{t-s} u(s), \quad t<s,
$$

where $\lambda_{t}$ is defined for $t<0$ as

$$
\lambda_{t}=\frac{1-e^{t}}{\left(1+e^{t}\right)^{2 n-1}} .
$$

We can now prove that the two limits in (2.4) are equal. As already noted, $\nu_{f}(x) \geq N_{f}(x)$. In the other direction we can take for instance $s=t+1$ in (2.6) to obtain the estimate

$$
U(t) \leq\left(1-\lambda_{-1}\right) U(t+1)+\lambda_{-1} u(t+1),
$$

whence

$$
\frac{U(t)}{t} \geq\left(1-\lambda_{-1}\right) \frac{U(t+1)}{t}+\lambda_{-1} \frac{u(t+1)}{t}, \quad t<0 .
$$

Letting $t$ tend to $-\infty$ we see that $N_{f}(x) \geq \nu_{f}(x)$.

For any given $f, q \in \operatorname{PSH}(\omega)$ we define

$$
\varphi_{\tau}(x)=\inf _{t}\left[u_{f}(x, t)-\tau \operatorname{Re} t\right], \quad x \in \omega, \tau \geq 0 .
$$

In view of our convention that $u_{f}(x, t)=+\infty$ if $(x, t) \notin \Omega_{q}$, the infimum operation acts effectively only over those $t$ that satisfy $\operatorname{Re} t<-q(x)$. The function $\tau \mapsto-\varphi_{\tau}(x)$ is the Fenchel transform of $\mathbb{R} \ni t \mapsto u_{f}(x, t)$. We assume all the time that $e^{-q(x)}$ does not exceed the distance $d_{\omega}(x)$ from $x$ to the boundary of $\omega$, so that $u_{f}$ is well defined. The function $(x, t) \mapsto$ $u_{f}(x, t)-\tau \operatorname{Re} t$ is plurisubharmonic in $\Omega_{q}$ and independent of the imaginary part of $t$. Therefore the minimum principle of Kiselman [1978] can be applied and yields that $\varphi_{\tau}$ is plurisubharmonic in $\omega$.

EXAMPle. Let us look at the simplest example: $f(x)=\log |x|, x \in \mathbb{C}^{n}$. We choose $q=0$ and form $U_{f}(x, t)=\log \left(e^{t}+|x|\right)$ for $t<q(x)=0$. Then $\varphi_{\tau}(x)=\inf _{t<0}\left(U_{f}(x, t)-\tau t\right)$ can be calculated explicitly: it is $\varphi_{\tau}(x)=$ $(1-\tau) \log |x|+C_{\tau}$ for $0 \leq \tau<1$, where $C_{\tau}$ is a constant which depends on the parameter $\tau$, and $\varphi_{\tau}(x)=\log (1+|x|)$ for $\tau \geq 1$. Thus the Lelong number of $\varphi_{\tau}$ at the origin is $\max (1-\tau, 0)$ for all $\tau \geq 0$.

There is no apparent reason why the Lelong number of the plurisubharmonic function $\varphi_{\tau}$ at $x$ should be a function of $\nu_{f}(x)$ and $\tau$; it could as well depend in some other way on the behavior of $f$ near $x$. However, it turns out that the simple formula for the Lelong number of $\varphi_{\tau}$ in the example holds quite generally: 
Theorem 2.1 (Kiselman [1979]). Let $f, q \in \operatorname{PSH}(\omega)$ with $q \geq-\log d_{\omega}$. Define $\varphi_{\tau}$ by (2.2) and (2.7). Then $\varphi_{\tau} \in \operatorname{PSH}(\omega)$. If $\nu_{q}(x)=0$, then the Lelong number of $\varphi_{\tau}$ is

$$
\nu_{\varphi_{\tau}}(x)=\max \left(\nu_{f}(x)-\tau, 0\right)=\left(\nu_{f}(x)-\tau\right)^{+}, \quad x \in \omega, \tau \geq 0 .
$$

We can also use the function $U_{f}$ instead of $u_{f}$ in the construction; the proof is the same. If $\tau<0$, then of course $\nu_{\varphi_{\tau}}(x)=+\infty$.

We shall give a simplified proof of Theorem 2.1 under the slightly stronger hypothesis that $q(x)>-\infty$. This is quite enough for the applications we have in mind. (As soon as $\omega \neq \mathbb{C}^{n}$, we must indeed have $q>-\infty$ everywhere.)

Lemma 2.2. With $f$ and $\varphi_{\tau}$ as in Theorem 2.1 we have

$$
\nu_{\varphi_{\tau}}(x) \geq \nu_{f}(x)-\tau \text {. }
$$

Proof. We first note that by the definition of $\varphi_{\tau}$, we have for any $t^{\prime}$,

$$
\varphi_{\tau}(y) \leq u_{f}\left(y, t^{\prime}\right)-t^{\prime} \tau
$$

We now take the mean value of both sides of (2.9) when $y$ varies over the sphere $x+e^{t} S$, which gives

$$
u_{\varphi_{\tau}}(x, t)=f_{z \in S} \varphi_{\tau}\left(x+z e^{t}\right) \leq \int_{z \in S} f_{w \in S} f\left(x+z e^{t}+w e^{t^{\prime}}\right)-t^{\prime} \tau
$$

for all real numbers $t, t^{\prime}$. Here the double integral defines a measure of total mass 1 which is invariant under rotations around $x$ and has its support in the set

$$
x+e^{t} S+e^{t^{\prime}} S=\left\{x+z:\left|e^{t}-e^{t^{\prime}}\right| \leq|z| \leq e^{t}+e^{t^{\prime}}\right\} .
$$

When we apply this measure to $f$, the result is a weighted mean of $f$, which, since it is rotation invariant, is also a mean value of $u_{f}(x, s)$ for various choices of $s$. The values of $s$ that can occur are those which satisfy

$$
\left|e^{t}-e^{t^{\prime}}\right| \leq e^{s} \leq e^{t}+e^{t^{\prime}}=e^{t^{\prime \prime}},
$$

where the last equality serves to define the number $t^{\prime \prime}$. Since $u_{f}(x, s)$ is increasing in $s$, the mean value cannot exceed $u_{f}\left(x, t^{\prime \prime}\right)$. Thus (2.10) yields

$$
u_{\varphi_{\tau}}(x, t) \leq f_{z \in S} f_{w \in S} f\left(x+z e^{t}+w e^{t^{\prime}}\right)-t^{\prime} \tau \leq u_{f}\left(x, t^{\prime \prime}\right)-t^{\prime} \tau .
$$

(The corresponding formula for $U_{\varphi_{\tau}}$ is even simpler to prove.) The only interesting choice is $t=t^{\prime}$, so that $t^{\prime \prime}=t+\log 2$. Thus

$$
\frac{u_{\varphi_{\tau}}(x, t)}{t} \geq \frac{u_{f}(x, t+\log 2)}{t}-\tau, \quad t<0,
$$

and letting $t$ tend to $-\infty$ we get the desired conclusion.

Lemma 2.3. With $f$ and $\varphi_{\tau}$ as in Theorem 2.1, take a number $\tau>\nu_{f}(x)$. Assume that $q(x)>-\infty$. Then $\varphi_{\tau}(x)>-\infty$. In particular, $\nu_{\varphi_{\tau}}(x)=0$. 
Proof. Since $\nu_{f}(x)<\tau<+\infty, f$ is not equal to $-\infty$ identically in a neighborhood of $x$. The value $\varphi_{\tau}(x)$ is the infimum of the convex function $u_{f}(x, t)-t \tau$ of the real variable $t$ when $t$ varies in the interval $]-\infty,-q(x)[$. This interval is by hypothesis bounded from the right. Moreover, by the choice of $\tau$, the function is strictly decreasing when $t \ll 0$. Thus its infimum is finite.

Proof of Theorem 2.1, assuming that $q(x)$ is finite. The proof consists of the following steps (cf. Kiselman [1992]). First we note that $\varphi_{\tau}$ is a concave function of $\tau$ with $\varphi_{0}=f$. Therefore $\nu_{\varphi_{\tau}}(x)$ is a convex function of $\tau$ taking the value $\nu_{f}(x)$ for $\tau=0$, for the Lelong number is, as we have seen, a linear function of $\varphi$, the limit of $u_{\varphi}(x, t) / t$. Second we see from Lemma 2.2 that $\nu_{\varphi_{\tau}}(x) \geq \nu_{f}(x)-\tau$. Third we know from Lemma 2.3 (if $\nu_{f}(x)$ is finite) that $\nu_{\varphi_{\tau}}(x)=0$ if $\tau>\nu_{f}(x)$. The only convex function of $\tau$ which has these properties is $\tau \mapsto\left(\nu_{f}(x)-\tau\right)^{+}$.

3. The Hörmander-Bombieri theorem and the integrability index. The fundamental tool which will provide us with analytic sets is the Hörmander-Bombieri theorem:

TheOREM 3.1. Let $\omega$ be a pseudoconvex open set in $\mathbb{C}^{n}$, and let $\varphi \in$ $\operatorname{PSH}(\omega)$. For every $a \in \omega$ such that $e^{-\varphi} \in L_{\text {loc }}^{2}(a)$ there exists a holomorphic function $h \in \mathcal{O}(\omega)$ such that $h(a)=1$ and

$$
\int_{\omega}|h|^{2} e^{-2 \varphi}\left(1+|z|^{2}\right)^{-3 n} d \lambda_{2 n}(z)<+\infty .
$$

Here $L_{\text {loc }}^{2}(a)$ denotes the set of all functions that are square integrable in some neighborhood of the point $a$.

For the proof see Hörmander [1990, Theorem 4.4.4]. Denote by $\mathcal{O}(\omega, \varphi)$ the set of all holomorphic functions $h$ in $\omega$ which satisfy condition (3.1) for a given function $\varphi$. The intersection

$$
V(\varphi)=\bigcap_{h \in \mathcal{O}(\omega, \varphi)} h^{-1}(0)
$$

is an intersection of zero sets of holomorphic functions, and therefore itself an analytic set. Define

$$
I(\varphi)=\left\{a \in \omega: e^{-\varphi} \notin L_{\mathrm{loc}}^{2}(a)\right\} .
$$

With this notation the theorem says that $V(\varphi) \subset I(\varphi)$. It is, however, obvious that $I(\varphi) \subset V(\varphi)$.

In view of this theorem it is natural to measure the singularity of a plurisubharmonic function $\varphi$ at a point $a$ by its integrability index $\iota_{\varphi}(a)$ :

$$
\iota_{\varphi}(a)=\inf _{t>0}\left[t: e^{-\varphi / t} \in L_{\mathrm{loc}}^{2}(a)\right] .
$$


Let $\Phi$ be an arbitrary subset of $\operatorname{PSH}(\omega)$ and $\kappa$ a functional on $\Phi$ in the sense that there is given a function $\kappa_{\varphi}: \omega \rightarrow[0,+\infty]$ for every $\varphi \in \Phi$. We introduce a notation for the superlevel sets of such functionals:

$$
E_{c}^{\kappa}(\varphi)=\left\{a \in \omega: \kappa_{\varphi}(a) \geq c\right\}, \quad c \geq 0 .
$$

The superlevel sets of the integrability index are analytic varieties. In fact, by the definition of $\iota$,

$$
E_{c}^{\iota}(\varphi) \subset I(\varphi / t) \subset E_{t}^{\iota}(\varphi), \quad 0<t<c .
$$

Using the Hörmander-Bombieri theorem we see that

$$
E_{c}^{\iota}(\varphi) \subset V(\varphi / t) \subset E_{t}^{\iota}(\varphi), \quad 0<t<c .
$$

We now note that by the definition of the superlevel set, the intersection of all $E_{t}^{\iota}(\varphi)$ when $t$ varies in the interval $0<t<c$ is just $E_{c}^{\iota}(\varphi)$, so that

$$
E_{c}^{\iota}(\varphi)=\bigcap_{0<t<c} V(\varphi / t), \quad c \geq 0
$$

Suppose that $\kappa$ is a functional which is comparable to the integrability index in the sense that the inequality

$$
s \iota_{\varphi}(x) \leq \kappa_{\varphi}(x) \leq t \iota_{\varphi}(x), \quad \varphi \in \Phi, x \in \omega,
$$

holds for some positive constants $s$ and $t$. Then there is of course a relation between the superlevel sets of the two functionals:

$$
E_{t c}^{\kappa}(\varphi) \subset E_{c}^{\iota}(\varphi) \subset E_{s c}^{\kappa}(\varphi), \quad \varphi \in \Phi .
$$

If we know that $E_{t c}^{\kappa}(\varphi)=E_{s c}^{\kappa}(\varphi)$, then $E_{t c}^{\kappa}(\varphi)$ equals $E_{c}^{\iota}(\varphi)$ and so is an analytic variety. Of course functions which admit such an interval of constancy in their superlevel sets are very special. But we shall see in the next section that for a set of plurisubharmonic functions such intervals of constancy can appear quite naturally.

We now ask whether the Lelong number is comparable to the integrability index in the sense of (3.5). The answer is well known, but will be quoted here for convenience.

TheOREM 3.2. If $\varphi \in \operatorname{PSH}(\omega)$, where $\omega \subset \mathbb{C}^{n}$, and $\nu_{\varphi}(a) \geq n$, then $e^{-\varphi} \notin L_{\mathrm{loc}}^{2}(a)$. Thus $E_{n}^{\nu}(\varphi) \subset I(\varphi) \subset V(\varphi)$. In terms of the integrability index we have $\nu_{\varphi}(x) \leq n \iota_{\varphi}(x)$.

Proof. This result is contained in Skoda [1972, Proposition 7.1], but it is easy to give a proof using the function $U=U_{\varphi}$ defined by (2.3). If $\nu_{\varphi}(a) \geq n$, then the slope of $t \mapsto U(a, t)$ at $-\infty$ is at least $n$, and we have

$$
U(a, t) \leq U\left(a, t_{0}\right)+n\left(t-t_{0}\right), \quad t \leq t_{0},
$$


for some $t_{0}$. Rewriting this in terms of $\varphi$ we see that

$$
\varphi(z) \leq \varphi\left(z_{0}\right)+\log \left(\frac{|z-a|^{n}}{\left|z_{0}-a\right|^{n}}\right), \quad|z-a| \leq\left|z_{0}-a\right|,
$$

for a suitable point $z_{0}$ on the sphere $|z-a|=e^{t_{0}}$, or equivalently,

$$
e^{-2 \varphi(z)} \geq e^{-2 \varphi\left(z_{0}\right)} \frac{\left|z_{0}-a\right|^{2 n}}{|z-a|^{2 n}}
$$

where the right-hand side is a non-integrable function near $a$.

In the other direction we have:

TheOREM 3.3. If $\varphi \in \operatorname{PSH}(\omega)$ has a finite value at a point $a \in \omega$, then $e^{-\varphi} \in L_{\mathrm{loc}}^{2}(a)$.

For a proof of this result, see Hörmander [1990, Theorem 4.4.5]. The theorem says that $I(\varphi)$ is contained in the polar set $P(\varphi)=\varphi^{-1}(-\infty)$ of $\varphi$, i.e. $I(\varphi) \subset P(\varphi)$.

Combining Theorems 3.1, 3.2 and 3.3 we see that

$$
E_{n c}^{\nu}(\varphi) \subset V(\varphi / c) \subset P(\varphi), \quad c>0 .
$$

A stronger result in the same direction is

TheOREM 3.4. If $\nu_{\varphi}(a)<1$, then $e^{-\varphi} \in L_{\text {loc }}^{2}(a)$. Thus $I(\varphi) \subset E_{1}^{\nu}(\varphi)$. Also, $\iota_{\varphi}(x) \leq \nu_{\varphi}(x)$.

For the proof see Skoda [1972, Proposition 7.1].

Combining Theorems 3.1, 3.2 and 3.4 we get

THEOREM 3.5. The Lelong number $\nu$ is comparable to the integrability index $\iota$ in the sense of (3.5), more precisely,

$$
\iota_{\varphi}(x) \leq \nu_{\varphi}(x) \leq n \iota_{\varphi}(x), \quad \varphi \in \operatorname{PSH}(\omega), x \in \omega \subset \mathbb{C}^{n},
$$

and

$$
E_{n c}^{\nu}(\varphi) \subset V(\varphi / c) \subset E_{c}^{\nu}(\varphi) \subset V(n \varphi / c), \quad \varphi \in \operatorname{PSH}(\omega), c>0 .
$$

These inequalities are sharp.

This result is the basis for the analyticity theorems that we shall state. However, the weaker result (3.7) is often sufficient.

That the comparison in (3.8) between the Lelong number and the integrability index cannot be improved follows from simple examples:

EXAMPLE 3.6. The function

$$
f(z)=\max \left(\log \left|z_{1}\right|^{a}, \log \left|z_{2}\right|^{b}\right), \quad z \in \mathbb{C}^{2},
$$


has integrability index $\iota_{f}(0)=a b /(a+b)$ and Lelong number $\nu_{f}(0)=$ $\min (a, b)$. Thus

$$
\left.\left.\frac{\nu_{f}(0)}{\iota_{f}(0)}=\frac{a+b}{\max (a, b)}=\frac{a^{-1}+b^{-1}}{\max \left(a^{-1}, b^{-1}\right)} \in\right] 1,2\right] .
$$

A little more generally, if we take $1 \leq k \leq n$ and positive numbers $a_{1}, \ldots, a_{k}$ and define

$$
f(z)=\max _{1 \leq j \leq k}\left(\log \left|z_{j}\right|^{a_{j}}\right), \quad z \in \mathbb{C}^{n},
$$

then $\iota_{f}(0)=\left(\sum a_{j}^{-1}\right)^{-1}$ (cf. Lemma 5.5 below) and $\nu_{f}(0)=\min a_{j}$, so that

$$
\frac{\nu_{f}(0)}{\iota_{f}(0)}=\frac{\sum a_{j}^{-1}}{\max a_{j}^{-1}} \in[1, n] .
$$

(These formulas hold if we define $a_{j}^{-1}=0$ for $j=k+1, \ldots, n$.) Clearly, the quotient $\nu_{f} / \iota_{f}$ can assume all values in the closed interval $[1, n]$ (we allow $k=1$ and $k=n$ ). Thus (3.8) is sharp.

\section{Analyticity theorems for sets of plurisubharmonic functions.} Let $\omega$ be an open set in $\mathbb{C}^{n}$ and $\Phi$ an arbitrary subset of $\operatorname{PSH}(\omega)$. Let $\kappa$ be a functional on $\Phi$ which is comparable to the integrability index in the sense that (3.5) holds for some positive constants $s$ and $t$. Then from (3.6) and (3.7) we get

$$
\bigcap_{\varphi \in \Phi} E_{t c}^{\kappa}(\varphi) \subset \bigcap_{\varphi \in \Phi} E_{c}^{\iota}(\varphi) \subset \bigcap_{\varphi \in \Phi} E_{s c}^{\kappa}(\varphi) \subset \bigcap_{\varphi \in \Phi} P(\varphi) .
$$

It is convenient to introduce a notation for these sets:

$$
E_{c}^{\kappa}(\Phi)=\bigcap_{\varphi \in \Phi} E_{c}^{\kappa}(\varphi)
$$

for any functional $\kappa$. If we define the value of the functional on the whole set $\Phi$ as

$$
\kappa_{\Phi}(x)=\inf _{\varphi \in \Phi} \kappa_{\varphi}(x),
$$

then $E_{c}^{\kappa}(\Phi)$ is just the superlevel set of $\kappa_{\Phi}$. We can also define the polar set of $\Phi$ as

$$
P(\Phi)=\bigcap_{\varphi \in \Phi} P(\varphi) .
$$

With this notation we can write (4.1) as

$$
E_{t c}^{\kappa}(\Phi) \subset E_{c}^{\iota}(\Phi) \subset E_{s c}^{\kappa}(\Phi) \subset P(\Phi) .
$$

THEOREM 4.1. Let $\kappa$ be a functional on a subset $\Phi$ of $\operatorname{PSH}(\omega)$ which is comparable to the integrability index in the sense that (3.5) holds for some positive constants $s$ and $t$. If the superlevel sets $E_{c}^{\kappa}(\Phi)$ are independent of 
c over an interval of sufficiently large logarithmic length, viz. if $E_{s c}^{\kappa}(\Phi)=$ $E_{t c}^{\kappa}(\Phi)$, then $E_{s c}^{\kappa}(\Phi)$ is an analytic variety. A little more generally, if $Y$ is an analytic subset of $\omega$ and $Y \cap E_{s c}^{\kappa}(\Phi)=Y \cap E_{t c}^{\kappa}(\Phi)$, then $Y \cap E_{s c}^{\kappa}(\Phi)$ is analytic.

Proof. Since the result is local, we can assume $\omega$ to be pseudoconvex. The inclusions (4.2) then show that $E_{s c}^{\kappa}(\Phi)=E_{c}^{\iota}(\Phi)$, which is the set of common zeros of a family of holomorphic functions in $\omega$; see (3.4). Similarly, $Y \cap E_{s c}^{\kappa}(\Phi)=Y \cap E_{c}^{\iota}(\Phi)$.

TheOREM 4.2. Let $\omega, \kappa$ and $\Phi$ be as in Theorem 4.1, and assume in addition that $\kappa$ is positively homogeneous, i.e., $\varphi \in \Phi$ and $t>0$ implies $t \varphi \in \Phi$ and $\kappa_{t \varphi}(a)=t \kappa_{\varphi}(a)$. Let $Y$ be an analytic subset of $\omega$, and let $X \subset Y$. Assume that

$$
\inf _{x \in X} \kappa_{\varphi}(x)>0 \quad \text { for all } \varphi \in \Phi,
$$

and

(4.4) for each $x \in Y \backslash X$ there exists a function $\varphi \in \Phi$ such that $\kappa_{\varphi}(x)=0$.

Then $X$ is an analytic set.

Proof. Define $\psi=\varphi / \varepsilon_{\varphi}$, where $\varepsilon_{\varphi}=\inf _{x \in X} \kappa_{\varphi}(x)>0$, and let $\Psi$ be the set of all functions $\psi$ obtained in this way. Then $\kappa_{\psi}(x) \geq 1$ for every $\psi \in \Psi$ and every $x \in X$. Using the notation for superlevel sets we can write this as

$$
X \subset E_{1}^{\kappa}(\Psi) \subset E_{c}^{\kappa}(\Psi)
$$

for all $c$ with $0 \leq c \leq 1$.

On the other hand, if $x \in Y \backslash X$, then by (4.4) there is a $\varphi \in \Phi$ such that $\kappa_{\varphi}(x)=0$. Thus $\psi=\varphi / \varepsilon_{\varphi}$ is in $\Psi$ and $\kappa_{\psi}(x)=\kappa_{\varphi}(x) / \varepsilon_{\varphi}=0$. So $x \notin E_{c}^{\kappa}(\psi), c$ being any positive number. Thus

$$
x \notin E_{c}^{\kappa}(\Psi)=\bigcap_{\psi \in \Psi} E_{c}^{\kappa}(\psi) .
$$

Therefore $E_{c}^{\kappa}(\Psi) \cap Y \subset X$ for every $c>0$. Combining this with the first part of the proof we see that $E_{c}^{\kappa}(\Psi) \cap Y=X$ for all $c$ satisfying $0<c \leq 1$. Hence $E_{c}^{\kappa}(\Psi) \cap Y$ is constant for these $c$ and Theorem 4.1 yields that $X$ is analytic.

A particular case of Theorem 4.2 is when we can associate with a given function or current a family of plurisubharmonic functions on which our functional takes values that we can control. The following result is of this character. It also holds for functionals which have only a loose connection to the integrability index or the Lelong number; more precisely, functionals which are zero at the same time as the integrability index in a semiuniform way: 
Theorem 4.3. Let $\Phi=\left\{\varphi_{\alpha}: \alpha \in A\right\}$ be a set of plurisubharmonic functions in an open set $\omega$, and let $\kappa$ be a functional on $\Phi$ which is weakly comparable to the integrability index in the sense that for every $\varepsilon>0$ there is a $\delta>0$ such that

$$
\varphi \in \Phi, \iota_{\varphi}(x)<\delta \text { implies } \kappa_{\varphi}(x)<\varepsilon,
$$

and

$$
\varphi \in \Phi, \kappa_{\varphi}(x)=0 \quad \text { implies } \iota_{\varphi}(x)=0 .
$$

Suppose that the values $\kappa_{\varphi_{\alpha}}(x)$ are given by $\kappa_{\varphi_{\alpha}}(x)=G(H(x), \alpha)$ for some functions $G:[0,+\infty] \times A \rightarrow[0,+\infty]$ and $H: \omega \rightarrow[0,+\infty]$. Assume that $c \mapsto$ $G(c, \alpha)$ is increasing, and that there exists a number $c_{0}$ such that $G\left(c_{0}, \alpha\right)$ $>0$ for all $\alpha \in A$. Finally, suppose that for every $c<c_{0}$ there is an $\alpha \in A$ such that $G(c, \alpha)=0$. Then the superlevel set $\left\{x: H(x) \geq c_{0}\right\}$ is analytic.

Proof. We shall apply Theorem 4.2 to $X=\left\{x: H(x) \geq c_{0}\right\}$. First we note that

$$
\inf _{x \in X} \kappa_{\varphi_{\alpha}}(x)=\inf _{x \in X} G(H(x), \alpha) \geq G\left(c_{0}, \alpha\right)=\varepsilon_{\alpha}>0
$$

for any $\alpha \in A$. Hence $\iota_{\varphi_{\alpha}}(x) \geq \delta_{\alpha}>0$, which means that (4.3) holds for $\iota$. Next, if $x \notin X$, then $c=H(x)<c_{0}$ and there is an $\alpha$ such that $G(c, \alpha)=0$. We get

$$
\kappa_{\varphi_{\alpha}}(x)=G(H(x), \alpha)=G(c, \alpha)=0 ;
$$

hence also $\iota_{\varphi_{\alpha}}(x)=0$, so that (4.4) holds for $\iota$. Thus Theorem 4.2, applied to $\iota$, shows that $X$ is analytic.

This theorem contains the classical theorem of Siu [1974]. For if we let $\kappa_{\varphi}(x)=\nu_{\varphi}(x), A=\left[0, c_{0}\left[, G(c, \alpha)=(c-\alpha)^{+}, H(x)=\nu_{f}(x)\right.\right.$, and define $\varphi_{\alpha}$ as

$$
\varphi_{\alpha}(x)=\inf _{t}\left[u_{f}(x, t)-t \alpha:(x, t) \in \Omega_{q}\right], \quad x \in \omega, \alpha \in\left[0, c_{0}[,\right.
$$

then by Theorem 2.1,

$$
\nu_{\varphi_{\alpha}}(x)=\left(\nu_{f}(x)-\alpha\right)^{+}=G(H(x), \alpha) .
$$

The function $G(c, \alpha)=(c-\alpha)^{+}$satisfies the hypotheses of Theorem 4.3, so it follows that the superlevel set $\left\{x: \nu_{f}(x) \geq c_{0}\right\}$ is an analytic variety. The singularities of the $\varphi_{\alpha}$ are the same as those of $f$, but attenuated to some degree as shown by the formula. This attenuation is the reason behind their usefulness in proving Siu's theorem and the explanation for the title of this paper.

5. A refined Lelong number. In analogy with the mean value and supremum over a sphere, we can define the mean value and supremum over a polycircle. Given a function $f \in \operatorname{PSH}(\omega)$, let 


$$
\begin{aligned}
& v(x, y)=v_{f}(x, y)=\int_{\left|z_{j}\right|=\mid e^{y_{j} \mid}} f(x+z) \\
&=(2 \pi)^{-n} \int_{0}^{2 \pi} d s_{1} \ldots \int_{0}^{2 \pi} d s_{n} f\left(x_{1}+e^{y_{1}+i s_{1}}, \ldots, x_{n}+e^{y_{n}+i s_{n}}\right) ; \\
& V(x, y)=V_{f}(x, y)=\sup _{\left|z_{j}\right|=\left|e^{y_{j}}\right|} f(x+z) \\
&=\sup _{\left|z_{j}\right|=1} f\left(x_{1}+z_{1} e^{y_{1}}, \ldots, x_{n}+z_{n} e^{y_{n}}\right) .
\end{aligned}
$$

Using well-known properties of plurisubharmonic functions we see that $v$ and $V$ are plurisubharmonic functions of the variables $(x, y) \in \omega \times \mathbb{C}^{n}$ if $\operatorname{Re} y_{j} \ll 0$. To be precise, they are plurisubharmonic in the open set

$$
\Omega_{0}=\left\{(x, y) \in \omega \times \mathbb{C}^{n}: x+z \in \omega \text { for all } z \text { with }\left|z_{j}\right| \leq\left|e^{y_{j}}\right|\right\} .
$$

If $\omega$ is pseudoconvex, then so is $\Omega_{0}$. Indeed, if $d_{\omega}$ now denotes the distance to $\mathbb{C}^{n} \backslash \omega$ measured by the norm $\|z\|_{\infty}=\max _{j}\left|z_{j}\right|$, then $\Omega_{0}$ is the set of all $(x, y) \in \omega \times \mathbb{C}^{n}$ such that $\operatorname{Re} y_{j}<\log d_{\omega}(x)$. We note that $(x, y) \mapsto-\log d_{\omega}(x)+\max _{j} \operatorname{Re} y_{j}$ is plurisubharmonic in $\omega \times \mathbb{C}^{n}$, which implies that $\Omega_{0}$, as the subset of $\omega \times \mathbb{C}^{n}$ where this function is negative, is pseudoconvex.

We fix the domain of definition of $v$ and $V$ to be an open pseudoconvex set $\Omega$ contained in $\omega \times \mathbb{C}^{n}$ such that

$$
(x, y) \in \Omega,\left|z_{j}\right| \leq\left|e^{y_{j}}\right| \text { implies } \quad x+z \in \omega
$$

(this amounts to $\Omega \subset \Omega_{0}$ ) and

$$
(x, y) \in \Omega, \operatorname{Re} y_{j}^{\prime} \leq \operatorname{Re} y_{j} \quad \text { implies }\left(x, y^{\prime}\right) \in \Omega .
$$

Then we know that $v, V \in \operatorname{PSH}(\Omega)$. We define them as $+\infty$ outside $\Omega$. Moreover, $y \mapsto v(x, y), V(x, y)$ are convex and coordinatewise increasing.

Sometimes it is necessary to assume also

For every $x \in \omega$ and every $y \in \mathbb{R}_{+}^{n}$ there is a number $t_{x, y}<+\infty$ such that $(x, t y) \in \Omega$ implies $\operatorname{Re} t<t_{x, y}$.

Here $\mathbb{R}_{+}^{n}$ is the set of all vectors $y \in \mathbb{R}^{n}$ with $y_{j}>0$ for every $j$. If $\omega$ is bounded, (5.5) of course follows from (5.3).

Definition 5.1. Let $f \in \operatorname{PSH}(\omega)$ and define $v$ by (5.1). Then the function

$$
\mathbb{R}_{+}^{n} \ni y \mapsto \nu_{f}(x, y)=\lim _{t \rightarrow-\infty} \frac{v(x, t y)}{t}, \quad x \in \omega,
$$

will be called the refined Lelong number of $f$ at the point $x$. In the closed cone defined by $y_{j} \geq 0$ we define it by means of continuity: $\nu_{f}(x, y)=$ $\lim _{z \rightarrow y, z_{j}>0} \nu_{f}(x, z)$, and we set $\nu_{f}(x, y)=-\infty$ if some $y_{j}$ is negative. 
It is clear that $\nu_{f}(x, y)$ is positively homogeneous in $y: \nu_{f}(x, t y)=$ $t \nu_{f}(x, y)$ for all $y \in \mathbb{R}^{n}$ and all $t>0$. Moreover, it is concave as a function of $y \in \mathbb{R}^{n}$ for fixed $x$. In fact, $y \mapsto \nu_{f}(x, y)$ is the asymptotic function of the concave function $y \mapsto-v_{f}(x,-y)$ on $\mathbb{R}_{+}^{n}$. Its values are finite on $\mathbb{R}_{+}^{n}$ except when $f$ is equal to $-\infty$ in a whole neighborhood of $x$.

EXAMPLE. If $f(z)=\max _{j}\left(\log \left|z_{j}\right|^{a_{j}}\right)$ with positive numbers $a_{j}$, then $v(0, y)=\max _{j}\left(a_{j} y_{j}\right)$ for $y \in \mathbb{R}_{+}^{n}$, and the refined Lelong number at the origin is $\nu_{f}(0, y)=\min _{j}\left(a_{j} y_{j}\right), y \in \mathbb{R}_{+}^{n}$, a simple concave function of $y$.

Comparing $v$ and $V$ is analogous to comparing $u$ and $U$. We use Harnack's inequality $(2.5)$ with $m=2$ repeatedly to get

$$
f(z) \leq \prod_{1}^{n} \frac{1-\left|z_{j}\right| / r_{j}}{1+\left|z_{j}\right| / r_{j}} \underset{\left|y_{k}\right|=r_{k}}{f} f(y)
$$

provided $f \leq 0$ is plurisubharmonic in a neighborhood of the polydisk with radii $r_{1}, \ldots, r_{n}$. This gives

$$
V(x, z) \leq \prod_{1}^{n} \frac{1-e^{z_{j}-y_{j}}}{1+e^{z_{j}-y_{j}}} v(x, y), \quad z_{j}<y_{j} \leq \log r_{j} .
$$

If now $f$ is only plurisubharmonic, we can apply this inequality to the function $f-V(\cdot, y) \leq 0$. In complete analogy with (2.6) we can write

$$
V(x, z) \leq\left(1-\lambda_{z-y}\right) V(x, y)+\lambda_{z-y} v(x, y), \quad z_{j}<y_{j},
$$

where now

$$
\lambda_{z}=\prod_{1}^{n} \frac{1-e^{z_{j}}}{1+e^{z_{j}}}, \quad z_{j}<0
$$

and it follows that the refined Lelong number can be defined using $V$ as well as $v$.

One motivation for this variant of the Lelong number is in the study of blow-ups of plurisubharmonic functions. To give an idea of how blowups work, let us look at a simple example. Consider the mapping $T(x)=$ $\left(x_{1}, x_{1} x_{2}\right)$ from $\mathbb{C}^{2}$ into $\mathbb{C}^{2}$. The pull-back under this mapping of the algebraic set

is

$$
Y=\left\{y \in \mathbb{C}^{2}: y_{2}=0\right\} \cup\left\{y \in \mathbb{C}^{2}: y_{2}=y_{1}^{2}\right\}
$$

$X=T^{-1}(Y)=\left\{x \in \mathbb{C}^{2}: x_{1}=0\right\} \cup\left\{x \in \mathbb{C}^{2}: x_{2}=0\right\} \cup\left\{x \in \mathbb{C}^{2}: x_{1}=x_{2}\right\}$.

We see that in $Y$ the two branches are tangent to each other at the origin, whereas the irreducible branches of $X$ intersect transversally.

Now use the mapping $T$ to blow up a plurisubharmonic function at the origin. The blow-up is $g=T^{*} f, g\left(x_{1}, x_{2}\right)=f(T(x))=f\left(x_{1}, x_{1} x_{2}\right)$, and it 
turns out that the usual Lelong number of $g$ at the origin is equal to $\nu_{f}(0, y)$ with $y=(1,2)$.

More generally, a blow-up transforms the refined Lelong number by a linear transformation: if

$$
g(x)=T^{*} f(x)=f(T(x))=f\left(x_{1}^{m_{11}} x_{2}^{m_{12}} \ldots x_{n}^{m_{1 n}}, \ldots, x_{1}^{m_{n 1}} x_{2}^{m_{n 2}} \ldots x_{n}^{m_{n n}}\right)
$$

with $\operatorname{det}\left(m_{j k}\right) \neq 0$, then

$$
\nu_{g}(0, y)=\nu_{f}(0, z), \quad \text { where } z_{j}=\sum m_{j k} y_{k} .
$$

In fact,

$$
\begin{aligned}
V_{g}(0, y) & =\sup _{\left|x_{j}\right|=e^{y_{j}}} f\left(x_{1}^{m_{11}} x_{2}^{m_{12}} \ldots x_{n}^{m_{1 n}}, \ldots, x_{1}^{m_{n 1}} x_{2}^{m_{n 2}} \ldots x_{n}^{m_{n n}}\right) \\
& =\sup _{t_{j}} f\left(e^{z_{1}+i t_{1}}, \ldots, e^{z_{n}+i t_{n}}\right)=V_{f}(0, z)
\end{aligned}
$$

if we put

$$
x_{1}^{m_{j 1}} \ldots x_{n}^{m_{j n}}=\exp \left(\sum m_{j k} y_{k}+i \sum m_{j k} s_{k}\right)=\exp \left(z_{j}+i t_{j}\right) .
$$

Not only is $\left|x_{1}^{m_{j 1}} \ldots x_{n}^{m_{j n}}\right|=\exp z_{j}$, but also all arguments of $x_{1}^{m_{j 1}} \ldots x_{n}^{m_{j n}}$ occur independently if $\operatorname{det}\left(m_{j k}\right) \neq 0$.

Proposition 5.2. Let $f \in \operatorname{PSH}(\omega), x \in \omega$, and put $\mathbf{1}=(1,1, \ldots, 1) \in$ $\mathbb{R}^{n}$. Then $\nu_{f}(x, \mathbf{1})=\nu_{f}(x)$.

Proof. The ball of radius $e^{t}$ is contained in the polydisk with all radii equal to $e^{t}$, which in turn is contained in the ball of radius $e^{t} \sqrt{n}$. Therefore

$$
U(x, t) \leq V(x, t, \ldots, t) \leq U\left(x, t+\frac{1}{2} \log n\right) .
$$

Dividing by $t$ and letting $t$ tend to $-\infty$ we get the result.

Proposition 5.3. If $f \in \operatorname{PSH}(\omega), x$ is a point in $\omega$, and $y \in \mathbb{R}_{+}^{n}$, then

$$
\left(\min y_{j}\right) \nu_{f}(x) \leq \nu_{f}(x, y) \leq\left(\max y_{j}\right) \nu_{f}(x) .
$$

Proof. As already noted, the function $y \mapsto V(x, y)$ is increasing in each variable, so the result follows from the simple fact that the polydisk with radii $y_{j}$ contains the polydisk with all radii equal to $\min y_{j}$ and is contained in the polydisk with all radii equal to $\max y_{j}$. By homogeneity $\nu_{f}\left(x,\left(\max y_{j}\right) \mathbf{1}\right)=\left(\max y_{j}\right) \nu_{f}(x, \mathbf{1})$ and similarly for the minimum. Then we use Proposition 5.2 to replace $\nu_{f}(x, \mathbf{1})$ by $\nu_{f}(x)$.

Proposition 5.4. Fix $a \in \mathbb{C}^{n}$ and let $f$ be any plurisubharmonic function in a neighborhood of $a$. If there exists a $y \in \mathbb{R}^{n}$ with all $y_{j} \geq 0$ such that $\nu_{f}(a, y) \geq \sum y_{j}$, then $e^{-f} \notin L_{\mathrm{loc}}^{2}(a)$. 
Proof. Without loss of generality we can assume that $a=0$, that $f$ is defined in a neighborhood of the unit polydisk and that $V(0,0)=0$. If there is a $y \in \mathbb{R}_{+}^{n}$ such that $\nu_{f}(0, y) \geq q \sum y_{j}$ for some positive number $q$, then

$\sup _{\left|z_{k}\right| \leq \exp \left(t y_{k}\right)} f(z)=V(0, t y)=V(0, t y)-V(0,0) \leq q t \sum y_{j}, \quad$ for all $t \leq 0$,

which implies that

$$
f(z) \leq\left(\max _{k} \frac{1}{y_{k}} \log \left|z_{k}\right|\right) q \sum y_{j}, \quad\left|z_{j}\right| \leq 1
$$

or equivalently,

$$
e^{-f(z)} \geq \min _{k}\left|z_{k}\right|^{-q \Sigma_{j} y_{j} / y_{k}}=\min _{k}\left|z_{k}\right|^{-1 / c_{k}}, \quad\left|z_{j}\right| \leq 1,
$$

on putting $c_{k}=y_{k} /\left(q \sum y_{j}\right)$. Then $\sum c_{k}=1 / q$, and if $q \geq 1$ it follows from Lemma 5.5 below that $e^{-f}$ does not belong to $L_{\text {loc }}^{2}(0)$.

Now in general we only know that there is a $y$ with $y_{j} \geq 0$ satisfying the condition. Then by continuity there are vectors $y^{\prime} \in \mathbb{R}_{+}^{n}$ satisfying $\nu_{f}\left(0, y^{\prime}\right) \geq$ $q \sum y_{j}^{\prime}$ with $q$ arbitrarily close to 1 . We can now let $q$ tend to 1 and again conclude that $e^{-2 f}$ is not integrable; see the next lemma.

LEMMA 5.5. Let $c_{1}, \ldots, c_{n}$ be positive numbers and define $q=1 / \sum c_{k}$. The function

$$
\mathbb{C}^{n} \ni z \mapsto \min _{k}\left|z_{k}\right|^{-1 / c_{k}}
$$

is in $L_{\mathrm{loc}}^{2}(0)$ if and only if $q<1$. When $q<1$, the square of its $L^{2}$ norm over the unit polydisk is $\pi^{n} /(1-q)$, which tends to $+\infty$ as $q \nearrow 1$.

Proof. A direct calculation.

The converse of Proposition 5.4 does not hold in general, as can be seen from the simple example

$$
f(z)=\frac{3}{2} \log \left|z_{1}-z_{2}\right|, \quad z \in \mathbb{C}^{2} .
$$

Here

$$
\nu_{f}(0, y)=\frac{3}{2} \min y_{j} \leq \frac{3}{4}\left(y_{1}+y_{2}\right), \quad y \in \mathbb{R}^{2} .
$$

But $\exp (-f)=\left|z_{1}-z_{2}\right|^{-3 / 2} \notin L_{\text {loc }}^{2}(0)$.

However, for functions of $\left(\left|z_{1}\right|, \ldots,\left|z_{n}\right|\right)$ there is a converse:

Proposition 5.6. Let $f$ be a plurisubharmonic function of $\left(\left|z_{1}\right|, \ldots,\left|z_{n}\right|\right)$ in a neighborhood of the origin, and assume that there is a number $q$ satisfying $0<q<1$ such that $\nu_{f}(0, y) \leq q \sum y_{j}$ for all $y \in \mathbb{R}_{+}^{n}$. Then $e^{-f} \in L_{\mathrm{loc}}^{2}(0)$.

For the proof we need a lemma on the behavior of the asymptotic function of a convex function. 
LEMMA 5.7. Let $f$ be plurisubharmonic in a neighborhood of the closed unit polydisk. Suppose $q$ is a positive number such that $\nu_{f}(0, y) \leq q \sum y_{j}$ for all $y \in \mathbb{R}_{+}^{n}$, and take any $p>q$. Then there exists a constant $C$ such that $v(0, y) \geq p \sum y_{j}-C$ for all $y \in \mathbb{R}^{n}$ with $y_{j} \leq 0$.

Proof. We may assume that $v(0,0)=0$; then $v(0, y) \leq 0$ for all $y$ with $y_{j} \leq 0$. Let $\Gamma$ denote the simplex

$$
\Gamma=\left\{y \in \mathbb{R}^{n}: y_{j} \leq 0, \sum y_{j}=-1\right\} .
$$

Take a number $s$ with $p>s>q$. The hypothesis that $\nu_{f}(0, y) \leq q \sum y_{j}$ implies that for every $y \in \Gamma$ there is a constant $C_{y}$ such that $v(t y) \geq$ $-s t-C_{y}$; the question is whether $C_{y}$ can be chosen independently of $y \in \Gamma$. Choose finitely many points $y^{k} \in \Gamma$ such that for every $y \in \Gamma$ there is a $k$ and a point $z \in \Gamma$ such that

$$
y^{k}=\left(1-\frac{s}{p}\right) z+\frac{s}{p} y .
$$

Then by the convexity of $y \mapsto v(0, y)$,

$$
v\left(0, t y^{k}\right) \leq\left(1-\frac{s}{p}\right) v(0, t z)+\frac{s}{p} v(0, t y) \leq \frac{s}{p} v(0, t y), \quad t \geq 0 .
$$

There is a constant $C$ such that $v\left(t y^{k}\right) \geq-s t-C$ for all $k$ and all $t \geq 0$. Hence

$$
v(0, t y) \geq \frac{p}{s} v\left(0, t y^{k}\right) \geq-p t-C, \quad t \geq 0, y \in \Gamma,
$$

which is just what we wanted to prove.

Proof of Proposition 5.6. It is enough to consider $f$ satisfying the conditions of Lemma 5.7. By the lemma there exists a number $p<1$ and a finite constant $C$ such that $v(0, y) \geq p \sum y_{j}-C$ for all $y$ with $y_{j} \leq 0$. This means that $f(z) \geq p \log \left|z_{1} \ldots z_{n}\right|-C$ and hence

$$
e^{-2 f(z)} \leq\left|z_{1} \ldots z_{n}\right|^{-2 p} e^{2 C}, \quad\left|z_{j}\right| \leq 1
$$

The right hand side is integrable.

The results proved so far imply that we can determine the integrability index from the refined Lelong number for functions of $\left(\left|z_{1}\right|, \ldots,\left|z_{n}\right|\right)$ :

THEOREM 5.8. Let $f$ be a plurisubharmonic function defined in a neighborhood of the origin in $\mathbb{C}^{n}$, and let $g$ be the function of $\left(\left|z_{1}\right|, \ldots,\left|z_{n}\right|\right)$ whose mean values over any polycircle with center at the origin agree with those of $f$ (thus $v_{g}(x, y)=v_{f}(x, y)$ and $\nu_{g}(0, y)=\nu_{f}(0, y)$ for all $\left.y\right)$. Then the integrability index of $g$ is

$$
\iota_{g}(0)=\sup \left[\nu_{f}(0, y): y_{j}>0, \sum y_{j}=1\right] \leq \iota_{f}(0) .
$$

Pr o of. Combine Propositions 5.4 and 5.6. 
6. Plurisubharmonic functions with attenuated singularities. With $\Omega$ satisfying (5.3) and (5.4) we define

$$
f_{y, \alpha}(x)=\inf _{t}[V(x, t y)-\alpha t], \quad y \in \mathbb{R}^{n}, \alpha \in \mathbb{R}, x \in \omega .
$$

(Note that $V(x, t y)=+\infty$ if $(x, t y) \notin \Omega$.) This function is plurisubharmonic in view of the minimum principle (Kiselman [1978]) since $(x, t) \mapsto V(x, t y)-$ $\alpha \operatorname{Re} t$ is plurisubharmonic and independent of the imaginary part of $t$. We note that the set $\{y:(x, y) \in \Omega\}$ is convex in view of (5.4), and that the set $\{t \in \mathbb{R}:(x, t y) \in \Omega\}$ is an interval. We shall study the Lelong number of $f_{y, \alpha}$. The first result is a generalization of Lemma 2.2 .

Lemma 6.1. Let $a, b \in \mathbb{R}_{+}^{n}$ and let $s>0$. Define $a \wedge b$ by $(a \wedge b)_{j}=$ $\min \left(a_{j}, b_{j}\right)$. Then $\nu_{f_{b, \alpha}}(x, a) \geq \nu_{f}(x, a \wedge s b)-\alpha s$. In particular, $\nu_{f_{a, \alpha}}(x, a) \geq$ $\nu_{f}(x, a)-\alpha$.

P r o o f. By the definition of $f_{b, \alpha}$ we always have $f_{b, \alpha}(x) \leq V(x, s b)-s \alpha$ for any $s \in \mathbb{R}$. We now take the supremum over the polycircle $\left\{x+z:\left|z_{j}\right|=\right.$ $\left.\exp a_{j}\right\}$, with an arbitrary $a \in \mathbb{R}^{n}$ :

$$
\begin{aligned}
V_{f_{b, \alpha}}(x, a) & =\sup _{\left|z_{j}\right|=e^{a_{j}}} f_{b, \alpha}(x+z) \leq \sup _{\left|z_{j}\right|=e^{a_{j}}}[V(x+z, s b)-s \alpha] \\
& =\sup _{\left|z_{j}\right|=e^{a_{j}}} \sup _{\left|w_{j}\right|=e^{s b_{j}}}[f(x+z+w)-s \alpha] \\
& =\sup _{\left|z_{j}\right|=e^{y_{j}}}[f(x+z)-s \alpha]=V_{f}(x, y)-s \alpha,
\end{aligned}
$$

where $y \in \mathbb{R}^{n}$ is defined by $e^{y_{j}}=e^{a_{j}}+e^{s b_{j}}$. Thus

$$
V_{f_{b, \alpha}}(x, a) \leq V_{f}(x, y)-s \alpha
$$

for every $a$ and every $s b$, with $y$ determined from the first two vectors. We now replace $a$ by $t a$ and $s$ by $t s$ with $t<0$. Then

$$
V_{f_{b, \alpha}}(x, t a) \leq V_{f}(x, t y(t))-t s \alpha,
$$

where now

$$
y_{j}(t)=\frac{1}{t} \log \left(e^{t a_{j}}+e^{t s b_{j}}\right)
$$

and we can estimate $y_{j}(t)$ from above and below, writing $c=a \wedge s b$ :

$$
c_{j} \geq y_{j}(t) \geq \frac{1}{t} \log 2+c_{j} .
$$

Dividing (6.2) by the negative number $t$ and letting $t$ tend to $-\infty$ we get the desired inequality, at least at each point of continuity of $y \mapsto \nu_{f}(x, y)$. If $f$ equals minus infinity near $x$, all Lelong numbers are equal to $+\infty$. If not, $V_{f}(x, \cdot)$ is a convex function which is real-valued, hence continuous, in the open set $y_{j}>0$, and $V_{f}(x, t y(t)) / t$ tends to its asymptotic function $\nu_{f}(x, c)$ as $t \rightarrow-\infty$. 
Lemma 6.2. Assume $\Omega$ satisfies (5.3), (5.4) and (5.5). If $\alpha>\nu_{f}(x, y)$, then $f_{y, \alpha}(x)>-\infty$; in particular, $\nu_{f_{y, \alpha}}(x, z)=0$ for all $z \in \mathbb{R}_{+}^{n}$.

Proof. The number $f_{y, \alpha}(x)$ is the infimum of $V(x, t y)-\alpha t$ over all real $t$ such that $(x, t y) \in \Omega$. The expression is a convex function of $t$ which is decreasing for $t \ll 0$. By (5.5), $t<t_{x, y}<+\infty$. Hence the infimum is finite.

Theorem 6.3. Let $\omega$ be open in $\mathbb{C}^{n}$ and let $\Omega$ be an open set in $\omega \times \mathbb{C}^{n}$ satisfying (5.3), (5.4) and (5.5). Let $f \in \operatorname{PSH}(\omega)$ and define $f_{y, \alpha}$ by (6.1). Then $\nu_{f_{y, \alpha}}(x, y)$ is determined by $\nu_{f}(x, y)$ and $\alpha$ :

$$
\nu_{f_{y, \alpha}}(x, y)=\left(\nu_{f}(x, y)-\alpha\right)^{+}, \quad x \in \omega, y \in \mathbb{R}_{+}^{n}, \alpha \geq 0 .
$$

Proof. The proof is analogous to that of Theorem 2.1. The function $\alpha \mapsto \nu_{\varphi_{\alpha}}(x, y)$ is convex, takes the value $\nu_{f}(x, y)$ for $\alpha=0$, and has the properties explained in Lemmas 6.1 and 6.2. This determines it uniquely.

\section{Analyticity theorems for the refined Lelong number}

THEOREM 7.1 (Analyticity theorem). For any $f \in \operatorname{PSH}(\omega)$, where $\omega$ is open in $\mathbb{C}^{n}$, any $y \in \mathbb{R}^{n}$ with $y_{j}>0$, and any real number $c$, the superlevel set

$$
E_{y, c}^{\nu}(f)=\left\{x \in \omega: \nu_{f}(x, y) \geq c\right\}
$$

of the refined Lelong number is an analytic variety in $\omega$.

This theorem was proved in April, 1986, and a proof was presented in June, 1986, at the conference Geometric and Quantitative Complex Analysis in Wuppertal. The theorem is stated without proof in Kiselman [1987]. A proof was published in Kiselman [1992]. The theorem generalizes Siu's theorem [1974] and is a special case of Demailly's analyticity theorem [1987].

Proof of Theorem 7.1. The functional $\kappa_{\varphi}(x)=\nu_{\varphi}(x, y)$ is comparable to the integrability index (Theorem 3.5 and Proposition 5.3) and is positively homogeneous in $\varphi$, so we can apply Theorem 4.3, letting $\Phi$ denote the set of all functions $f_{y, \alpha}$ with $\alpha \geq 0$ and using the information in Theorem 6.3.

It is tempting to try to prove an analyticity theorem for numbers that are defined by varying only some of the variables, thus defining

$$
V_{f, k}\left(x, y_{1}, \ldots, y_{k}\right)=\sup _{\left|z_{j}\right|=e^{y_{j}}} f\left(x_{1}+z_{1}, \ldots, x_{k}+z_{k}, x_{k+1}, \ldots, x_{n}\right)
$$

and

$\nu_{f, k}\left(x, y_{1}, \ldots, y_{k}\right)=\lim _{t \rightarrow-\infty} \frac{V_{f, k}\left(x, t y_{1}, \ldots, t y_{k}\right)}{t}, \quad x \in \omega,\left(y_{1}, \ldots, y_{k}\right) \in \mathbb{R}_{+}^{k}$. 
We could call $\nu_{f, k}$ a partial Lelong number, because it is the refined Lelong number of a restriction of $f$ to a $k$-dimensional subspace of $\mathbb{C}^{n}$. Evidently,

$$
V_{f, k}\left(x, y_{1}, \ldots, y_{k}\right) \leq V_{f}\left(x, y_{1}, \ldots, y_{n}\right)
$$

for all choices of $y_{k+1}, \ldots, y_{n} \in \mathbb{R}$, so that

$$
\nu_{f, k}\left(x, y_{1}, \ldots, y_{k}\right) \geq \nu_{f}\left(x, y_{1}, \ldots, y_{n}\right), \quad x \in \omega, y \in \mathbb{R}_{+}^{n},
$$

and

$$
\nu_{f, k}\left(x, y_{1}, \ldots, y_{k}\right) \geq \lim \nu_{f}\left(x, y_{1}, \ldots, y_{n}\right), \quad x \in \omega, y \in \mathbb{R}_{+}^{k},
$$

where the limit is for $y_{k+1}, \ldots, y_{n} \rightarrow+\infty$. It is natural to ask whether

$$
\nu_{f, k}\left(x, y_{1}, \ldots, y_{k}\right)=\lim \nu_{f}\left(x, y_{1}, \ldots, y_{n}\right), \quad x \in \omega, y \in \mathbb{R}_{+}^{k} .
$$

Clearly,

$$
V_{f, k}\left(x, y_{1}, \ldots, y_{k}\right)=\lim V_{f}\left(x, y_{1}, \ldots, y_{n}\right),
$$

where the limit is now taken as $y_{k+1}, \ldots, y_{n}$ tend to $-\infty$, but this is not enough to guarantee equality in (7.1). In general, there is no analyticity theorem for these partial Lelong numbers; see Wang [1991, Examples 4.2 and 4.3]. Under certain hypotheses, however, an analyticity theorem does hold; see Wang [1991, Theorems 4.10 and 4.11]. We shall now consider a very simple case of this kind, which moreover is of some geometric significance. We let $L\left(\mathbb{C}^{m}, \mathbb{C}^{n}\right)$ denote the space of all linear mappings from $\mathbb{C}^{m}$ into $\mathbb{C}^{n}$.

Theorem 7.2. Let $f \in \operatorname{PSH}(\omega)$. Define

$$
V_{f}^{\prime}(x, h, z, y)=\sup _{\substack{\left|x^{\prime}\right|=e^{y_{0}} \\\left|z_{j}^{\prime}\right|=e^{y_{j}}}} f\left(x+x^{\prime}+h\left(z+z^{\prime}\right)\right)
$$

for $(x, h, z, y) \in \omega \times L\left(\mathbb{C}^{m}, \mathbb{C}^{n}\right) \times \mathbb{C}^{m} \times \mathbb{R}^{1+m}$ and

$$
\nu_{f}^{\prime}(x, h, z, y)=\lim _{t \rightarrow-\infty} \frac{V_{f}^{\prime}(x, h, z, t y)}{t}
$$

for $(x, h, z, y) \in \omega \times L\left(\mathbb{C}^{m}, \mathbb{C}^{n}\right) \times \mathbb{C}^{m} \times \mathbb{R}_{+}^{1+m}$. Then the superlevel set

$$
E_{y, c}^{\nu^{\prime}}(f)=\left\{(x, h, z) \in \omega \times L\left(\mathbb{C}^{m}, \mathbb{C}^{n}\right) \times \mathbb{C}^{m}: \nu_{f}^{\prime}(x, h, z, y) \geq c\right\}
$$

is an analytic subvariety of $\omega \times L\left(\mathbb{C}^{m}, \mathbb{C}^{n}\right) \times \mathbb{C}^{m}$.

Proof. For $f \in \operatorname{PSH}(\omega)$ the function $F(x, h, z)=f(x+h(z))$ is plurisubharmonic in

$$
\Omega=\left\{(x, h, z) \in \omega \times L\left(\mathbb{C}^{m}, \mathbb{C}^{n}\right) \times \mathbb{C}^{m}: x+h(z) \in \omega\right\} .
$$

The refined Lelong number of this function is then well defined: we first take the supremum over a polycylinder

$$
\begin{aligned}
& V_{F}(X, Y)=V_{F}((x, h, z), Y)=\sup \left[F\left(X+X^{\prime}\right):\left|X_{j}^{\prime}\right|=e^{Y_{j}}\right] \\
= & \sup \left[f\left(x+x^{\prime}+\left(h+h^{\prime}\right)\left(z+z^{\prime}\right)\right):\left|x^{\prime}\right|=e^{y_{0}},\left|h_{j k}^{\prime}\right|=e^{y_{j k}},\left|z_{k}^{\prime}\right|=e^{y_{k}}\right]
\end{aligned}
$$


for

$$
X=(x, h, z) \in \Omega, \quad Y=\left(y_{0},\left(y_{j k}\right),\left(y_{k}\right)\right) \in \mathbb{R}^{1+m n+m},
$$

and then define the refined Lelong number as

$$
\nu_{F}(X, Y)=\lim _{t \rightarrow-\infty} \frac{V_{F}(X, t Y)}{t}, \quad X \in \Omega, Y \in \mathbb{R}_{+}^{1+m n+m} .
$$

Here $X=(x, h, z)$ is a point in $\Omega$, and $Y=\left(y_{0},\left(y_{j k}\right),\left(y_{k}\right)\right)$ denotes a direction in $\mathbb{R} \times \mathbb{R}^{m n} \times \mathbb{R}^{m}$. The $y_{j k}$ variables are those that correspond to the variation in $h$; the linear mapping $h^{\prime}$ is given by $h^{\prime}(z)_{j}=\sum h_{j k}^{\prime} z_{k}$ and we let the $h_{j k}^{\prime}$ vary under the constraints $\left|h_{j k}^{\prime}\right|=e^{y_{j k}}$. We shall see that if the $y_{j k}$ are large compared with $y_{0}$, then

$$
\nu_{F}((x, h, z), Y)=\nu_{f}^{\prime}(x, h, z, y) .
$$

Admitting this for a moment, we see that $E_{y, c}^{\nu^{\prime}}(f)=E_{Y, c}^{\nu}(F)$ if the vector $y=$ $\left(y_{0}, y_{1}, \ldots, y_{m}\right) \in \mathbb{R}^{1+m}$ is completed in a suitable way to $Y$. By Theorem 7.1 the set $E_{Y, c}^{\nu}(F)$ is analytic, which proves the theorem. The passage to the limit in (7.1) is therefore particularly simple: the value is constant for all large values of those variables that tend to plus infinity. In Wang [1991, Section 5] it is shown that there are other cases where the limit (7.1) gives an analyticity theorem for the partial Lelong number. It is, however, necessary to use polydisks with very small radii in some directions to get the result, which shows that the idea of using polydisks rather than balls is essential.

To prove (7.5) we shall compare the supremum in (7.3) with that in (7.4): in the first the mapping $h$ is fixed, in the second it varies. Obviously, $V_{F}((x, h, z), Y) \geq V_{f}^{\prime}(x, h, z, y)$ for all choices of $\left(y_{j k}\right)$, which gives $\nu_{F}((x, h, z), Y) \leq \nu_{f}^{\prime}(x, h, z, y)$.

On the other hand, if we write

$$
F\left(X+X^{\prime}\right)=f\left(x+x^{\prime}+\left(h+h^{\prime}\right)\left(z+z^{\prime}\right)\right)=f\left(x+x^{\prime \prime}+h\left(z+z^{\prime}\right)\right)
$$

with $x^{\prime \prime}=x^{\prime}+h^{\prime}\left(z+z^{\prime}\right)$, then in the definition of $V_{F}((x, h, z), t Y)$ (cf. (7.4)) we have

$$
\left|x^{\prime \prime}\right| \leq e^{t y_{0}}+\sum e^{t y_{j k}}\left(\left|z_{k}\right|+e^{t y_{k}}\right)=e^{t y_{0}(t)},
$$

where the last equality is a definition of $y_{0}(t)$. We get

$$
V_{F}((x, h, z), t Y) \leq V_{f}^{\prime}\left(x, h, z, t y_{0}(t), t y_{1}, \ldots, t y_{m}\right)
$$

and

$$
y_{0}(t)=\frac{1}{t} \log \left(e^{t y_{0}}+\sum e^{t y_{j k}}\left(\left|z_{k}\right|+e^{t y_{k}}\right)\right) \rightarrow y_{0} \quad \text { as } t \rightarrow-\infty,
$$


provided $0<y_{0}<y_{j k}$ and $y_{k}>0$ for all $j$ and $k$, yielding

$$
\begin{aligned}
\nu_{F}((x, h, z), Y) & =\lim _{t \rightarrow-\infty} \frac{V_{F}((x, h, z), t Y)}{t} \\
& \geq \lim _{t \rightarrow-\infty} \frac{V_{f}^{\prime}\left(x, h, z, t y_{0}(t), t y_{1}, \ldots, t y_{m}\right)}{t}=\nu_{f}^{\prime}(x, h, z, y) .
\end{aligned}
$$

Given positive numbers $y_{0}$ and $y_{1}, \ldots, y_{m}$ it is therefore enough to choose $y_{j k}$ such that $y_{j k}>y_{0}>0$. This means that the change in the variable $h$ is absorbed by the change in the variable $x$, and we can conclude that (7.5) holds if $y_{j k}>y_{0}>0$ and $y_{k}>0$.

Theorem 7.3. Define for $f \in \operatorname{PSH}(\omega)$,

$$
V_{f}^{\prime \prime}(x, h, y)=\sup _{\left|z_{j}\right| \leq e^{y_{j}}} f(x+h(z))
$$

for $(x, h, y) \in \omega \times \mathrm{GL}(n, \mathbb{C}) \times \mathbb{R}^{n}$ and

$$
\nu_{f}^{\prime \prime}(x, h, y)=\lim _{t \rightarrow-\infty} \frac{V_{f}^{\prime \prime}(x, h, t y)}{t}
$$

for $(x, h) \in \omega \times \mathrm{GL}(n, \mathbb{C})$ and $y \in \mathbb{R}_{+}^{n}$. Then every superlevel set

$$
E_{y, c}^{\nu^{\prime \prime}}(f)=\left\{(x, h) \in \omega \times \mathrm{GL}(n, \mathbb{C}): \nu_{f}^{\prime \prime}(x, h, y) \geq c\right\}
$$

of $\nu_{f}^{\prime \prime}(x, h, y)$ is an analytic subset of $\omega \times \operatorname{GL}(n, \mathbb{C})$.

Proof. We compare the supremum in (7.3) with that in (7.6). In the first, $x$ varies, in the second $x$ is fixed. Since $h$ is now invertible, the variation in $x$ can be absorbed by the variation in $z$, so that $V_{f}^{\prime}(x, h, 0, y)$ can be compared with $V_{f}^{\prime \prime}(x, h, y)$ as in the previous proof. Explicitly, we first note that we have $V_{f}^{\prime \prime}\left(x, h, y_{1}, \ldots, y_{n}\right) \leq V_{f}^{\prime}\left(x, h, 0, y_{0}, \ldots, y_{n}\right)$ for all real $y_{0}, y_{1}, \ldots, y_{n}$, which gives $\nu_{f}^{\prime \prime}(x, h, y) \geq \nu_{f}^{\prime}\left(x, h, 0, y_{0}, y\right)$ for all $y \in$ $\mathbb{R}_{+}^{n}, y_{0} \in \mathbb{R}_{+}$. For the other direction we look at the supremum defining $V_{f}^{\prime}\left(x, h, 0, t y_{0}, \ldots, t y_{n}\right)(\mathrm{cf.} .(7.3))$; we can write

$$
x+x^{\prime}+h\left(z^{\prime}\right)=x+h\left(h^{-1}\left(x^{\prime}\right)+z^{\prime}\right)=x+h\left(z^{\prime \prime}\right),
$$

where

$$
\left|z_{j}^{\prime \prime}\right|=\left|h^{-1}\left(x^{\prime}\right)_{j}+z_{j}^{\prime}\right| \leq\left\|h^{-1}\right\| e^{t y_{0}}+e^{t y_{j}}=e^{t y_{j}(t)},
$$

if we define

$$
y_{j}(t)=\frac{1}{t} \log \left(\left\|h^{-1}\right\| e^{t y_{0}}+e^{t y_{j}}\right) .
$$

If $y_{0}>y_{j}$, then $y_{j}(t)$ tends to $y_{j}$ as $t \rightarrow-\infty$. Therefore

$$
V_{f}^{\prime}\left(x, h, 0, t y_{0}, \ldots, t y_{n}\right) \leq V_{f}^{\prime \prime}\left(x, h, t y_{1}(t), \ldots, t y_{n}(t)\right),
$$

which in turn implies 
$\nu_{f}^{\prime}\left(x, h, 0, y_{0}, \ldots, y_{n}\right) \geq \nu_{f}^{\prime \prime}\left(x, h, y_{1}, \ldots, y_{n}\right)$

$$
y_{j}>0, y_{0}>\max \left(y_{1}, \ldots, y_{n}\right) \text {. }
$$

Given any $y \in \mathbb{R}_{+}^{n}$ we choose $y_{0}>\max y_{j}$ and then have $E_{y, c}^{\nu^{\prime \prime}}(f)=$ $E_{\left(y_{0}, y\right), c}^{\nu^{\prime}}(f)$. By Theorem 7.2 this is an analytic set.

Finally we shall study another kind of attenuation of the singularities. We define

$$
g_{\eta}(x)=\inf _{y}[V(x, y)-\operatorname{Re} \eta \cdot y:(x, y) \in \Omega], \quad x \in \omega, \eta \in \mathbb{R}^{n},
$$

where $\eta \cdot y=\eta_{1} y_{1}+\ldots+\eta_{n} y_{n}$.

Question. What is the refined Lelong number $\nu_{g_{\eta}}(x, y)$ of $g_{\eta}$ ? Is there a formula like (6.3)?

We shall collect a few results concerning the functions $g_{\eta}$ which are not enough to answer this question, but which nevertheless suffice to prove an analyticity theorem using them.

Lemma 7.4. Let $f \in \operatorname{PSH}(\omega)$ and define $g_{\eta}$ by (7.7). Then

$$
\begin{array}{ll}
\nu_{g_{\eta}}(x, a) \geq \nu_{f}(x, a \wedge b)-\eta \cdot b, & \eta \in \mathbb{R}^{n}, x \in \omega, a, b \in \mathbb{R}^{n} ; \\
\nu_{g_{\eta}}(x, a) \geq \sup _{b}\left(\nu_{f}(x, a \wedge b)-\eta \cdot b\right), & \eta \in \mathbb{R}^{n}, x \in \omega, a \in \mathbb{R}^{n} .
\end{array}
$$
that

Pr o of. We always have $g_{\eta} \leq f_{b, \alpha}$ if $\eta \cdot b=\alpha$. Using Lemma 6.1 we see

$$
\nu_{g_{\eta}}(x, a) \geq \nu_{f_{b, \alpha}}(x, a) \geq \nu_{f}(x, a \wedge b)-\alpha=\nu_{f}(x, a \wedge b)-\eta \cdot b .
$$

Lemma 7.5. Assume that $\Omega$ satisfies (5.3) and (5.4). Let $f \in \operatorname{PSH}(\omega)$ and define $g_{\eta}$ by (7.7). Take a point $x \in \omega$. If $a \in \mathbb{R}_{+}^{n}$ and $\nu_{f}(x, a)<c$, then there exists a vector $\eta \in \mathbb{R}^{n}$ such that $\eta \cdot a<c$ and $g_{\eta}(x)>-\infty$. In particular, $\nu_{g_{\eta}}(x, z)=0$ for all $z \in \mathbb{R}_{+}^{n}$.

Proof. If $\nu_{f}(x, a)<c$, there exists an affine minorant of $V$ on the straight line defined by $a$ : if we take a number $t_{0}$ with $-t_{0}$ large enough, there is a number $b$ such that $\nu_{f}(x, a) \leq b<c$ and

$$
-\infty<V\left(x, t_{0} a\right)+b\left(t-t_{0}\right) \leq V(x, t a)
$$

for every $t \in \mathbb{R}$. By the Hahn-Banach theorem, we can find an affine minorant in the whole space extending this one, which means that we can find $\eta \in \mathbb{R}^{n}$ such that $\eta \cdot a=b<c$ and

$$
V\left(x, t_{0} a\right)+\eta \cdot\left(y-t_{0} a\right) \leq V(x, y)
$$

for every $y \in \mathbb{R}^{n}$. Consequently,

$$
-\infty<C=V\left(x, t_{0} a\right)-\eta \cdot\left(t_{0} a\right) \leq V(x, y)-\eta \cdot y
$$

and $-\infty<C \leq \inf _{y}(V(x, y)-\eta \cdot y)=g_{\eta}(x)$. 
TheOREM 7.6. Let $\omega$ be an open set in $\mathbb{C}^{n}$ and $\Omega$ an open set in $\omega \times \mathbb{C}^{n}$ satisfying (5.3) and (5.4). Let $g_{\eta}$ be given by (7.7). Then $G_{x, z}(\eta)=\nu_{g_{\eta}}(x, z)$ is a convex function of $\eta \in \mathbb{R}^{n}$, and its Fenchel transform

$$
\widetilde{G}_{x, z}(y)=\sup _{\eta}\left[\eta \cdot y-G_{x, z}(\eta)\right]=\sup _{\eta}\left[\eta \cdot y-\nu_{g_{\eta}}(x, z)\right], \quad y \in \mathbb{R}^{n},
$$

satisfies

$$
-\nu_{f}(x, y) \leq \widetilde{G}_{x, z}(-y) \leq-\nu_{f}(x, y \wedge z), \quad y, z \in \mathbb{R}_{+}^{n} .
$$

In particular, $\widetilde{G}_{x, z}(-y)=-\nu_{f}(x, y)$ when $y \leq z$. The function $(y, z) \mapsto$ $\widetilde{G}_{x, z}(-y)$ is convex in $\mathbb{R}^{n} \times \mathbb{R}^{n}$.

Proof. From Lemma 7.4 we see that $-\nu_{g_{\eta}}(x, z) \leq-\nu_{f}(x, y \wedge z)+\eta \cdot y$, which immediately gives the inequality to the right in (7.8).

If $\nu_{f}(x, y)<c$, then by Lemma 7.5 there is an $\eta \in \mathbb{R}^{n}$ such that $\eta \cdot y<c$ and $\nu_{g_{\eta}}(x, z)=0$ for all $z \in \mathbb{R}^{n}$. Hence by definition $\widetilde{G}_{x, z}(-y) \geq-c$. Letting $c$ tend to $\nu_{f}(x, y)$, we get the inequality to the left in (7.8). This proves the theorem.

Can Theorem 4.3 be applied to prove Theorem 7.1 using instead the $g_{\eta}$ ? We do not know enough about the Lelong numbers of $g_{\eta}$ to do thatTheorem 4.3 requires an exact formula for the values of the functional. But the more flexible Theorem 4.2 will suffice. To see this, let $\Phi$ be the set of all functions $g_{\eta}$ with $\eta \cdot y<c$, where we define $g_{\eta}$ by (7.7). We first check the hypothesis (4.3) of Theorem 4.2. Lemma 7.4 yields

$$
\kappa_{g_{\eta}}(x)=\nu_{g_{\eta}}(x, y) \geq \nu_{f}(x, y)-\eta \cdot y \geq c-\eta \cdot y>0
$$

for any point $x \in X=E_{y, c}^{\nu}(f)$. Thus (4.3) holds. Next, if $\nu_{f}(x, y)<c$, then there is an $\eta$ such that $\eta \cdot y<c$, thus $g_{\eta} \in \Phi$, and $\kappa_{g_{\eta}}(x)=0$ (Lemma 7.5). This means that also (4.4) holds, and we can conclude again, now using Theorem 4.2 , that $X=E_{y, c}^{\nu}(f)$ is analytic.

\section{References}

L. Abrahamsson [1988], Microlocal Lelong numbers of plurisubharmonic functions, J. Reine Angew. Math. 388, 116-128.

J.-P. Demailly [1987], Nombres de Lelong généralisés, théorèmes d'intégralité et d'analyticité, Acta Math. 159, 153-169.

L. Hörmander [1990], Complex Analysis in Several Variables, North-Holland.

C. O. Kiselman [1978], The partial Legendre transformation for plurisubharmonic functions, Invent. Math. 49, 137-148.

- [1979], Densité des fonctions plurisousharmoniques, Bull. Soc. Math. France 107, 295304. 
C. O. Kiselman [1987], Un nombre de Lelong raffiné, in: Séminaire d'Analyse Complexe et Géométrie 1985-87, Faculté des Sciences de Tunis \& Faculté des Sciences et Techniques de Monastir, 61-70.

- [1992], La teoremo de Siu por abstraktaj nombroj de Lelong, in: Aktoj de Internacia Scienca Akademio Comenius, Vol. 1, Beijing, 56-65.

P. Lelong [1985], Les objets souples de l'analyse complexe, Exposition. Math. 3, 149-164.

Y.-T. Siu [1974], Analyticity of sets associated to Lelong numbers and the extension of closed positive currents, Invent. Math. 27, 53-156.

H. Skoda [1972], Sous-ensembles analytiques d'ordre fini ou infini dans $\mathbb{C}^{n}$, Bull. Soc. Math. France 100, 353-408.

X. Wang [1991], Analyticity theorems for parameter-dependent currents, Math. Scand. 69, 179-198.

DEPARTMENT OF MATHEMATICS

UPPSALA UNIVERSITY

P. O. BOX 480

S-751 06 UPPSALA, SWEDEN

E-mail: COK@MATH.UU.SE 